\title{
Grupos violentos en Centroamérica: la institucionalización de la violencia
}

\author{
Ailsa Winton
}

El artículo analiza las particularidades del entorno institucional en relación con la transformación y continuación de la violencia en la Centroamérica contemporánea. Sostiene que uno de los aspectos más significativos de esta violencia es la existencia de una amplia gama de instituciones y grupos violentos - estatales, privados, civiles, formales, informales, criminales- y que éstos están conectados de varias maneras y a diferentes escalas. Estas conexiones o nexos, y el arraigo sistémico de estos grupos, son lo que nos lleva a argumentar que la violencia y los grupos que la ejercen se están institucionalizando de una forma alarmante, y que esto constituye un elemento importante en el análisis de la violencia contemporánea en la región.

PALABRAS CLAVE: violencia, Centroamérica, grupos violentos, pandillas, institucionalización

\section{Violent Groups in Central America: the Institutionalization of Violence}

This article analyses the particularities of the institutional landscape in relation to the transformation and continuation of violence in contemporary Central America, arguing that one of the most significant aspects of this violence is the existence of a wide range of violent institutions and groups - state, private, civil, formal, informal, criminal-, and that these institutions are connected in various ways and at different scales. These connections or links, and the systemic introduction of these groups, that leads us to argue that violence and the groups that exercise it are becoming institutionalized to an alarming degree, something that constitutes an important element in the analysis of contemporary violence in the region.

KEYWORDS: violence, Central America, violent groups, gangs, institutionalization 


\section{PRESENTACIÓN}

$\mathrm{N}$ o obstante la complejidad y multidimensionalidad de la violencia, y la importancia de una gama de factores sociales y económicos en la perpetración de la misma dentro de un contexto "posconflicto" - o mejor dicho, "postransicional" (Cruz, 2003) - , el presente trabajo pretende argumentar que en el caso de la Centroamérica contemporánea los rasgos de los grupos violentos son los que tienen mayor influencia en las tendencias, manifestaciones y discrepancias de la violencia contemporánea en cuanto a su forma y severidad.

Se ha establecido que un rango de defectos políticos - la corrupción, la incompetencia, la brutalidad y la impunidad - tienen un efecto profundo sobre el aumento, transformación y continuación de la violencia en diferentes contextos, sobre todo en el llamado "posconflicto". Sin embargo, sería provechoso analizar con más detalle las particularidades del entorno institucional en relación con la transformación y continuación de la violencia en la Centroamérica contemporánea, sobre todo en términos de los impactos de estas transformaciones en un nivel local.

El análisis sostiene que uno de los aspectos más significativos de la violencia contemporánea - principalmente la urbana - es la amplia gama de instituciones y grupos violentos - estatales, privados, civiles, formales, informales, criminales-, y en especial las conexiones entre ellas en diferentes contextos. Estas conexiones, el cambiante poder inherente a estos grupos y su arraigo sistémico son los elementos que nos llevan a argumentar que la violencia y los grupos que la ejercen se están institucionalizando de una forma alarmante.

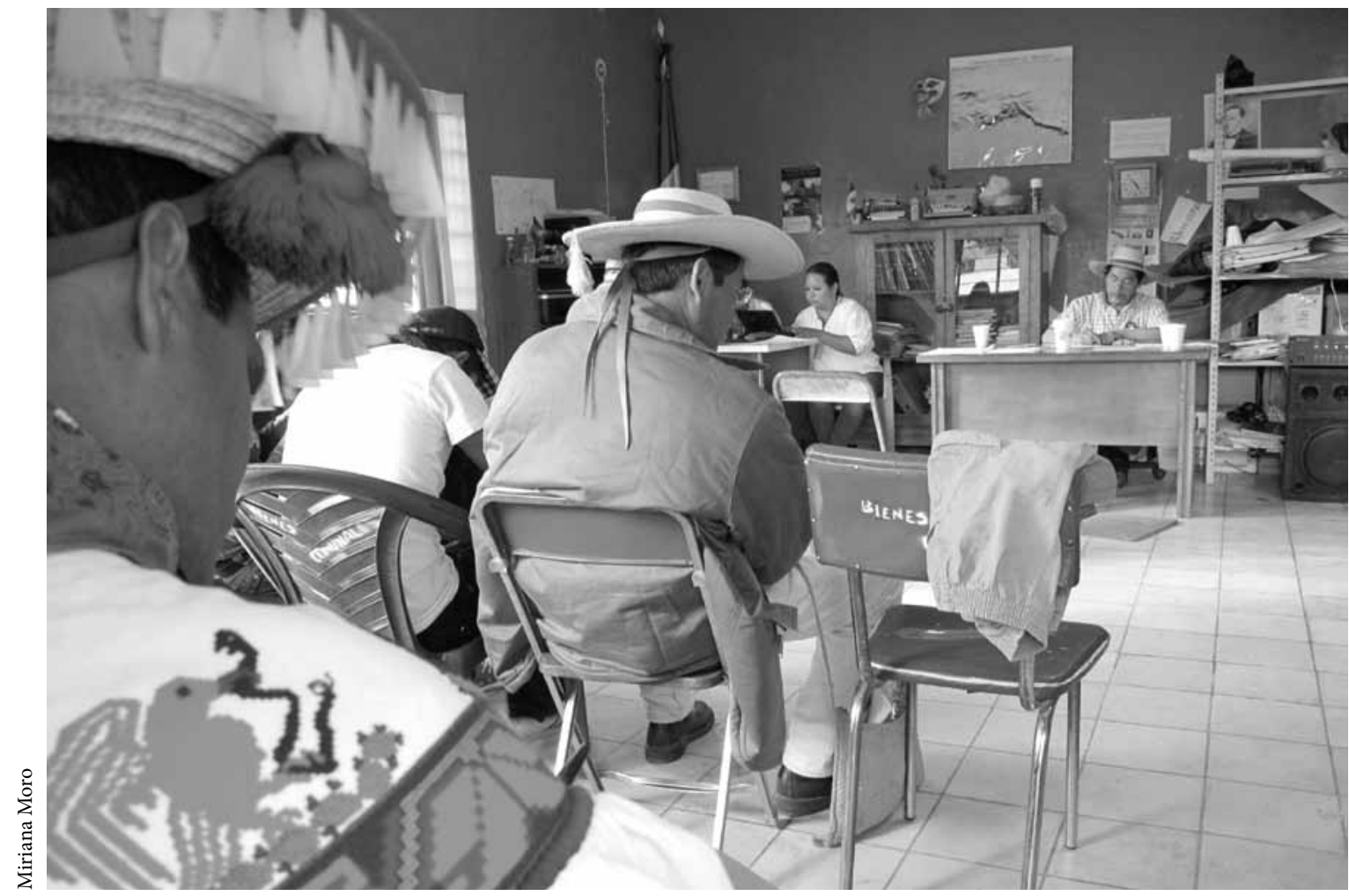

Trabajos al interior del Congreso Nacional Indígena en Mezcala, Jalisco, 2010. 


\section{LA HERENCIA DEL CONFLICTO POLÍTICO:VULNERABILIDAD ESTATAL E ILEGITIMIDAD INSTITUCIONAL}

La historia reciente de varios países de Centroamérica está marcada por conflictos civiles prolongados que han causado daño masivo a largo plazo, tanto en lo simbólico como en lo real, y que crearon vacíos locales de instituciones y asociaciones dentro de la región. La violencia política sostenida y la falta de seguridad legítima han contribuido en gran medida a la normalización de la violencia y a la formación de la espiral de violencia social que azota a muchos países centroamericanos, lo que da forma a una violencia más insidiosa y extendida que la de los conflictos políticos anteriores (Winton, 2004). ${ }^{1}$

La función, o bien disfunción del Estado, no remite tanto a las tensiones y amenazas a que éste es susceptible, como a su capacidad para contenerlas (véase Kirby, 2006): un Estado robusto no sólo proporciona a sus ciudadanos un medio de vida relativamente seguro, pues asimismo es capaz de frenar la aparición y escalada de la violencia organizada; en cambio, un Estado débil y vulnerable no nada más es incapaz de otorgar una ciudadanía significativa, sino que también deja vacante el espacio institucional-político que los grupos criminales organizados pueden ocupar. Un Estado vulnerable, cuyas instituciones, ya débiles, permiten el incremento de las actividades de grupos criminales organizados - actividades que

\footnotetext{
${ }^{1}$ Es interesante hacer notar que, entre tantas discusiones sobre la normalización de la violencia y la coexistencia problemática de violencia generalizada y democracia, el caso de la violencia contemporánea en Nicaragua es radicalmente distinto y se podría especular que se presenta un proceso de democratización más exitoso, donde, entre otros factores, la reforma de la policía, el sistema jurídico y el decomiso de armas contribuyeron, por lo menos en parte, a alcanzar niveles mucho más bajos de violencia -incluyendo la violencia de pandillas- en comparación con otros países, como Guatemala y El Salvador. Tampoco cabe duda de que los rasgos políticos radicalmente diferentes del conflicto armado en Nicaragua -en específico respecto del papel del Estado en el conflicto- tuvieron un impacto favorable en cuanto a la relativa estabilidad durante el periodo posterior y la eficaz ideología estatal.
}

luego potencian la degeneración del mismo aparato estatal-, un Estado que no es capaz de monopolizar el uso legítimo de la violencia, paralizado por la corrupción, es un Estado que carece de los fundamentos necesarios para manejar eficientemente los problemas que plantean estos grupos. Una vez que actores no estatales se institucionalizan, las instituciones del Estado se debilitan todavía más y se reduce de manera notable su capacidad para tomar acción. Como argumenta Mesa (2009), la incapacidad de los gobiernos para hacer frente a la violencia erosiona la legitimidad y la institucionalidad democrática y amenaza la estabilidad política. Por otro lado se plantea que son los problemas sociales y de gobernabilidad los que explican la intensificación del crimen organizado en la región (Mesa, 2009). Entramos, entonces, en un círculo vicioso, en el cual la falta de legitimidad estatal es tanto una causa como una consecuencia de la institucionalización de los grupos criminales. Lo anterior no debería dejar una impresión de un Estado débil pero benigno. Al contrario, uno de los aspectos más insidiosos en la continuación y transformación de la violencia en la región es la colusión recurrente del Estado en estos nexos violentos (véase abajo).

Tampoco sorprende que la desigual presencia institucional del Estado en el territorio se vea reflejada en una concentración desigual de luchas de poder a nivel local. Mientras no haya una presencia institucional legítima en una comunidad, el vacío creado necesariamente genera conflicto entre actores organizados que luchan para ocupar estos vacíos. El lado visible de estos grupos - criminales y/o violentossuele observarse en comunidades marginadas, caracterizadas por un lado por la "expulsión institucional"2 de su población (Zúñiga, 2007-2008) y por otro lado por ser víctimas de incursiones violentas de parte de las autoridades y de una fuerte estigmatización discursiva. Algunos comentaristas han denominado este proceso como "la criminalización de la pobreza".

\footnotetext{
2 Entendido esto como la negación de acceso al mercado y al Estado de cierta parte de la población.
} 
En un nivel individual, lo arriba mencionado, agravado por las reformas neoliberales a escala macro, en una situación en la cual el "proyecto de una sociedad nacional de ciudadanos [...] parece ser cada vez más agotado y desacreditado" (Rodgers, 2003), se ha argumentado que la fragmentación y polarización social permiten que otras agrupaciones - frecuentemente criminales - se conviertan en "un recurso para obtener una identidad reconocida” (Brinceño-León y Zubillaga, 2002: 27). En un nivel local, el espacio adquiere el rango de campo de batalla para reclamar una posición y pertenencia en la vida (Davids, en prensa), y la pandilla puede establecerse como una organización social. Como plantean Huhn et al. (2005), es posible que las maras constituyan una forma violenta de integración social, la cual crea un sistema de derecho anómico. Empezamos a ver, entonces, interconexiones institucionales respecto de la continuación y transformación de la violencia contemporánea.

\section{4}

\section{VIOLENCIA INSTITUCIONAL Y GRUPOS VIOLENTOS: LUCHAS DE PODERY COMPLICIDAD}

En este entorno de creciente ilegitimidad de instituciones estatales formales han surgido grupos alternativos con el fin de llenar - por varios motivos y en diversas formas- estos vacíos de poder y de estructuración sociopolítica. Se ha visto una continuación del miedo y la inseguridad de tiempos pasados, pero en el contexto de una nueva era de violencia en que ésta deja de ser terreno para los grupos tradicionales de poder, y se convierte cada vez más en "una opción para una multitud de actores en búsqueda de todo tipo de metas" (Kruijt y Koonings, 1999: 11). Cabe clarificar que los conceptos de "violencia institucionalizada" y de "institucionalización de grupos violentos" son distintos, pero se encuentran relacionados. Respecto de la existencia de estructuras institucionales responsables de un trato violento en contra de la población —en cercanía con la noción de "violencia estructural"-, la violencia institucional se puede entender de la siguiente forma:

si se puede hablar con propiedad de una "violencia institucionalizada" en América Latina es porque existe un tipo de violencia contra la población mayoritaria que está incorporada en el ordenamiento social, que es mantenida por las instituciones sociales y que se encuentra justificada y aun legalizada por el ordenamiento normativo de los regímenes imperantes (Zúñiga, 2008: 105).

En el presente trabajo la institucionalización de los grupos violentos, aunque de alguna manera vinculada a estos elementos estructurales, se entiende en términos de la forma en que una amplia gama de instituciones y grupos violentos - estatales, privados, civiles, formales, informales, criminales- están interconectados a diferentes escalas y del arraigo sistémico de estos grupos a nivel local. Este arraigo sistémico se relaciona con la permanencia y la consolidación de estos grupos en un contexto local de altos niveles de violencia institucional. Este aspecto se refiere, entonces, a la normalización de un grupo, a su inserción en la funcionalidad y gobernanza de un sistema local o hasta nacional. Estos grupos se vuelven institucionalizados en ausencia de controles necesarios y de estructuras alternativas, y con la presencia de los elementos necesarios para su permanencia.

Numerosos grupos e instituciones estatales, privados, cívicos y criminales están involucrados en la lucha por poder social, económico y político dentro de comunidades, pero también entre estas mismas entidades existe a veces cierta colusión en cuanto a la comisión de actos violentos, lo cual produce tanto una red múltiple de actores violentos - que son cada vez menos distinguibles- como una fusión entre diferentes tipos de violencia, con efectos deletéreos para las comunidades en las cuales operan estos grupos. El poder cambiante de estos grupos y su arraigo a nivel local y nacional son elementos fundamentales en su desarrollo.

La importancia creciente de grupos de narcotráfico es un ejemplo claro de la coalición y conflicto 
entre grupos y de la institucionalización de estas estructuras delictivas de un nivel transnacional a local. En casos extremos, estos grupos llegan a dominar la configuración institucional de la comunidad y crean una estructura tan arraigada que se percibe como normal. En los últimos años se ha visto un crecimiento alarmante en la presencia, poder e influencia de estos grupos criminales en la región, al igual que en otras de América Latina. ${ }^{3}$

En cuanto a la relación entre el Estado y grupos de narcotráfico, diversas fuentes, en distintos países de la región, documentan una filtración considerable de los grupos de crimen organizado a todos los estratos del aparato estatal, sobre todo en el tráfico y la venta de drogas, desde lo estratégico - por ejemplo, la corrupción de diputados- hasta lo operativo - la colusión de elementos de la policía local-. De esta forma, el Estado se convierte en un actor clave en lo que Cruz (2003: 53) llama "el lento desgaste de la legitimidad" de las instituciones estatales. Otro elemento importante en la institucionalización de los grupos de crimen organizado es la presencia de personas dispuestas a colaborar con ellos en comunidades marginadas. Actores cada vez más relevantes en este sentido son los pandilleros o los llamados mareros. El tema de la expansión e institucionalización de las pandillas juveniles se discute con más detalle a continuación, pero cabe mencionar en términos generales que la situación del narcotráfico se complica aún más por el involucramiento de otras organizaciones, como las pandillas, que han pasado a convertirse en peones de los carteles mexicanos, tanto en la distribución local de la droga como en labores de seguridad durante el tránsito (Mesa, 2009).

Como se mencionó arriba, el contexto posconflicto de muchos países en Centroamérica conlleva implicaciones institucionales en cuanto a la estabilidad

\footnotetext{
${ }^{3}$ Sobre Brasil, véase Dowdney (2003) y Leeds (1996). Sobre Colombia, Gaviria (1998), Pecaut (1999) y Moser y Mcllwaine (2004). Sobre Nicaragua, Rodgers (2006).
}

y viabilidad del sistema político formal. Un elemento fundamental en la transición democrática es la profunda reforma del aparato estatal en todas sus manifestaciones. Si las instituciones visibles no han logrado reformarse de una manera satisfactoria, a los grupos clandestinos del conflicto armado el proceso de democratización ni siquiera les ha afectado. La misma naturaleza del conflicto armado y la participación de la población en ello todavía tiene implicaciones para la ejecución de violencia organizada en una gran parte de la región.

Cruz (2003) argumenta que los regímenes autoritarios centroamericanos se prolongaron tanto porque la "sociedad civil" cooperaba con ellos. La colaboración "subalterna" fue un elemento muy importante en la aplicación de violencia en El Salvador y Guatemala, y son redes que podrían reactivarse con el pretexto del crimen y la violencia, formando nuevos movimientos incívicos, debilitando el proceso de democratización. Como indica Mesa (2009), durante los conflictos armados, escuadrones de la muerte de ultraderecha desarrollaron nexos con los cuerpos de seguridad y con grupos empresariales. El Estado no solamente toleró estas prácticas, sino que participó en ellas. Estos grupos utilizaron estrategias de sembrar el miedo y aterrorizar a la población, abandonando los cadáveres mutilados de víctimas de secuestro y tortura en la vía pública. Estas estructuras no fueron desmanteladas en la transición democrática. Al contrario, con el aumento del flujo de drogas en la región en los últimos años, estos grupos han adquirido poder de nuevo, estableciendo alianzas con las redes del crimen organizado y facilitando la inserción del mismo en el aparato estatal (Mesa, 2009). Éstos y otros grupos clandestinos, los llamados "poderes ocultos" o paralelos, siguen operando con impunidad. De hecho, para Reguillo (2005) el poder de la paralegalidad es mucho mayor que la ilegalidad misma, ya que la paralegalidad supone rechazar el sistema en sí y marca el comienzo de un pacto social alternativo.

Llamar a los sistemas de poder que operan fuera del sistema político poderes "paralelos" puede ser un 


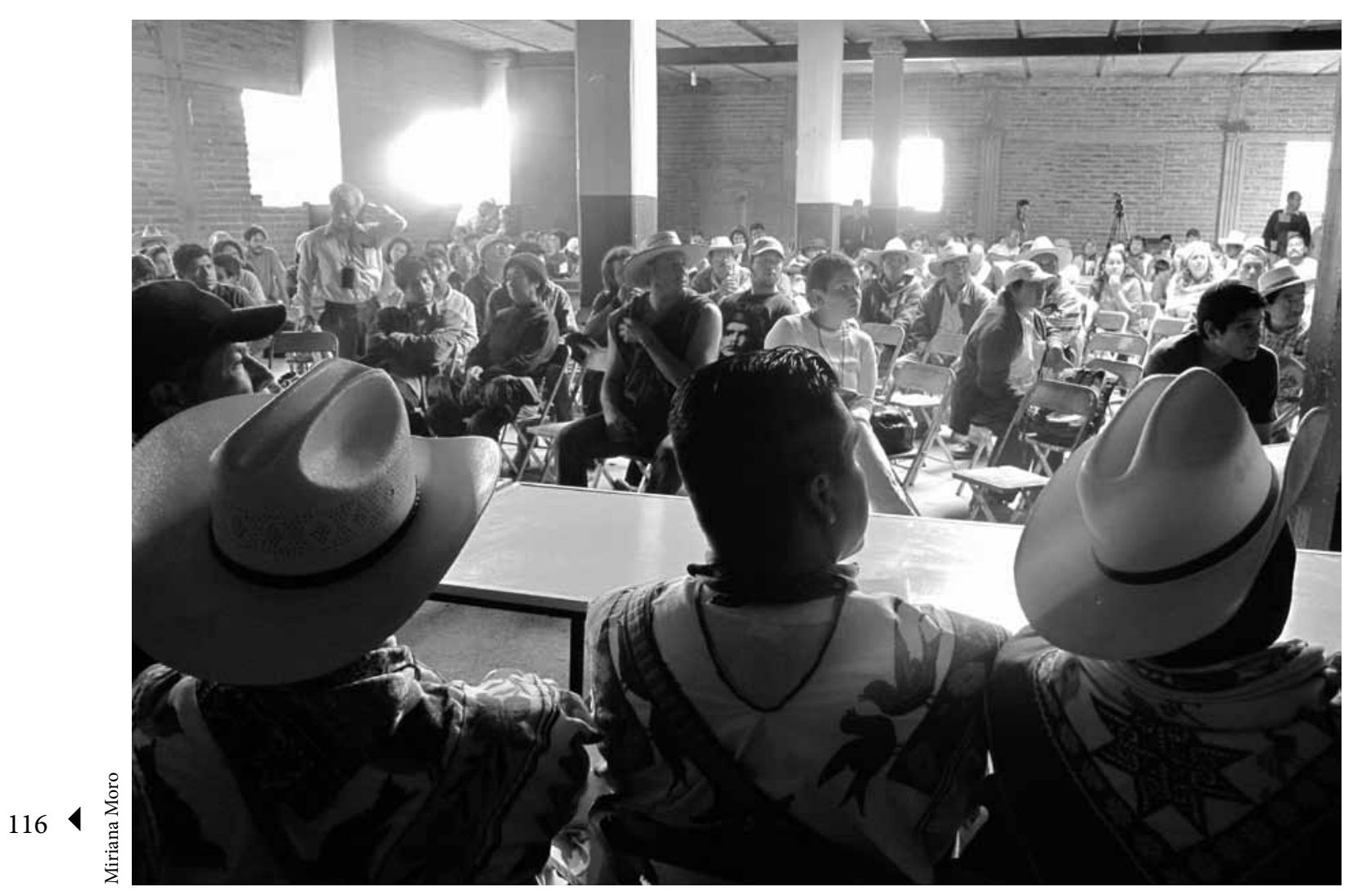

Plenaria en el Congreso Nacional Indígena, Mezcala, Jalisco, 2010.

término engañoso, ya que esto implica la falsa separación entre el poder "legítimo" y el "no legítimo", cuando en realidad, en el caso centroamericano, es precisamente la difuminación de la división entre lo estatal ("legítimo") y lo no estatal ("no legítimo") la que más perjudica el funcionamiento del mismo aparato estatal. En el caso de grupos de limpieza social, Cruz (2003: 51-52) argumenta que "el peor escenario es aquel en que las instituciones del Estado participan en este proceso de destrucción, al formar grupos de limpieza social, y motivar activamente a las personas a utilizar la violencia para defenderse".

Las matanzas extrajudiciales por parte de oficiales de la policía continúan siendo un problema en muchos contextos. En particular, las denuncias de "limpieza social" son comunes en Honduras y Guatemala, donde el Estado puede ser el autor, o actuar con frecuencia en colaboración con otros grupos informales integrados por ciudadanos de otros grupos "civiles", creando un nexo interinstitucional de actores violentos a nivel local (Moser y Winton, 2002). En Centroamérica, muchos de estos grupos tienen acuerdos tácitos con las autoridades locales - por ejemplo, "la sombra negra" en El Salvador-, lo cual implica no sólo el distanciamiento progresivo de las autoridades respecto de la población local, sino también la impunidad de éstos en actos ilegales de violencia extrema en contra de una amplia gama de personas "no deseadas". El tema de la justicia se complica todavía más cuando las autoridades locales, en vez de buscar la forma de llevar un control legítimo y legal a nivel local, se unen a estos grupos paralegales y buscan controlar el crimen y la violencia por medio de la justicia informal 
y arbitraria, que suele convertirse en la limpieza social. Curiosamente, mientras por un lado se ha documentado el apoyo de la policía por la justicia informal y la limpieza social (véase Mesa, 2009), esta misma institución muchas veces tiene un papel de cómplice con las pandillas (Medina y MateuGelabert, 2007; Zúñiga, 2007-2008). Es una situación en la cual la población se enfrenta a una ausencia total de protección de parte de las autoridades en un nivel local. En grupos de discusión llevados a cabo con jóvenes en comunidades marginadas en la ciudad de Guatemala, más que las pandillas o cualquier otro grupo, una institución constantemente identificada como una presencia negativa era la policía. Como argumenta Mesa (2009), la policía se convierte en parte del problema, en vez de ser parte de la solución.

La existencia de tales grupos clandestinos es de conocimiento común, si bien es difícil comprobar ya que todo hecho violento se atribuye a las pandillas de parte de la policía y los medios de comunicación (Huhn et al., 2005). Lo mismo sucede respecto de las acusaciones de acciones de limpieza social, ya que es fácil para los responsables hacer pasar a estos muertos como resultado de conflictos entre pandillas o por cuestiones relativas a la venta de drogas (Winton, 2004). El hecho de que actos de violencia extrema se registren en la comunidad sin que la gente sepa exactamente cuál de los muchos grupos "violentos" los cometió crea un ambiente de miedo y sospecha, ante una impunidad aparentemente total de los actores de esta violencia.

En el caso de una zona marginada en la ciudad de Guatemala se ha observado que desde el año 2000 estos hechos violentos han ido aumentando, sobre todo en contra de los jóvenes "pandilleros". En una pequeña colonia en esta zona se reportó en 2007 que 85 jóvenes fueron asesinados sólo en un año, muchas veces de manera brutal con técnicas semejantes a las de los escuadrones de muerte durante la época de conflicto armado. La aparición de cadáveres o cuerpos mutilados en las calles de la colonia genera terror entre la población local y una sensación de desampa- ro ante la impunidad con la que se cometen estos actos brutales. ${ }^{4}$ Sin embargo, la relación entre la "comunidad" por un lado, y los grupos violentos visibles - las pandillas, los narcotraficantes-y los grupos violentos no visibles - poderes ocultos, grupos de limpieza social, entre otros- por otro lado, es compleja. Los actores locales, lejos de estar separados del entorno local, forman parte del mismo. Aunque por una parte la justicia a propia mano se legitima frente a una situación de fracaso estatal, las víctimas de estas acciones son los mismos miembros de la comunidad. No son todos los jóvenes metidos en pandillas - representan una proporción mínima de la población joven-, y los que sí lo son, siguen siendo los hijos, los vecinos, los primos, los amigos de otros integrantes de la comunidad. De modo que las poblaciones que viven con estas complejas relaciones violentas en sus comunidades, sobre todo con la presencia de pandillas y todo lo que esto conlleva, experimentan una mezcla de miedo ante una violencia brutal y compasión por personas que son inseparables del entorno que las produjo (véase Medina y Mateu-Gelabert, 2007).

En términos generales, esta violencia brutal y arbitraria proviene de dos fenómenos relacionados: la falta de confianza en el Estado y la construcción del discurso de la violencia y la consiguiente deshumanización del "otro", del "no deseado". En primer lugar, cuando los ciudadanos han estado sujetos a una opresión estatal violenta, como es el caso, la confianza en el Estado como institución muy posiblemente sea baja. Dicha confianza puede verse aún más erosionada por un Estado posconflicto débil y corrupto, en el que los poderosos continúan protegidos por la impunidad. En especial, es pertinente una ausencia percibida de eficacia policial y judicial, lo cual no sólo convierte el control de la violencia en algo problemático, sino que directamente puede causar violencia en la forma de "vigilantismo" comunitario e individual. Las percepciones de la ley en sí están fuertemente relacionadas con las percepciones

\footnotetext{
${ }^{4}$ Entrevista en la ciudad de Guatemala, 12 de julio de 2007.
} 
del sistema judicial. Es significativo que en El Salvador, $40 \%$ de los adultos considera que es mejor hacer caso omiso de la ley si no se está de acuerdo con ella (Cruz, 1999).

Los linchamientos son un ejemplo de la posible continuación de los patrones de violencia que caracterizaban el conflicto armado (Moser y Winton, 2002). Según las estadísticas de la Misión de Verificación de las Naciones Unidas en Guatemala (Minugua), entre 1997 y 2001 hubo más de 817 víctimas de linchamientos, de los cuales 215 llegaron a la muerte (Fernández García, 2004). Estos hechos se llevaron a cabo en un ambiente de casi total impunidad, dada la amplia aprobación social de estas medidas arbitrarias. Se ha visto en años recientes que la justicia informal se ha institucionalizado cada vez más. Entonces, grupos locales de vigilancia vecinal tienen una presencia muy significativa en muchas comunidades pobres que carecen de servicios de seguridad estatales adecuados y, mientras se alimentan del miedo generalizado dentro de la comunidad, los vigilantes fomentan ese mismo miedo (Winton, 2004). En el contexto de África del Sur, Harris (2003) argumenta que el cambio en el discurso en relación con la violencia - de un acto político se convierte en un acto criminal - ha redefinido no sólo el tema de la violencia en sí, sino también los conceptos de legitimidad y justicia. Así, ciertas formas de violencia se criminalizan, pero simultáneamente se legitiman nuevos actos violentos, incluyendo "la lucha contra el crimen". En tal caso, el "vigilantismo reactivo" - el castigo de supuestos criminales - suele confluir con el "vigilantismo preventivo" - castigo de personas que podrían cometer crímenes-: las dos acciones se vuelven moralmente defendibles.

Como grupo social, son los jóvenes los que más sufren del castigo arbitrario a manos de los grupos de vigilancia. En la imaginación popular los jóvenes son criminalizados y por tanto son blancos legítimos en la lucha contra la delincuencia y la violencia. Esta deshumanización de la población joven se relaciona con la construcción de discursos sobre violencia. Estos temas de la legitimación de la violencia extrema, el discurso de violencia y las reacciones frente a una amenaza percibida se exploran aquí a través del caso específico de las pandillas juveniles en la región, las transformaciones que han experimentado como resultado de influencias institucionales y las reacciones que éstos han provocado para distintos actores.

\section{INSTITUCIONES E INTERVENCIONES VIOLENTAS: LA CAMBIANTE CARA DE LAS MARAS 5 EN CENTROAMÉRICA}

Las pandillas son, en parte, consecuencia de los factores arriba mencionados. Pero su relevancia para los propósitos de la presente discusión va más allá de sus antecedentes. Más bien, se argumenta que hoy en día son los factores institucionales los que tienen el impacto más fuerte sobre el funcionamiento de las pandillas en Centroamérica. Primero, los cambios en las políticas migratorias dieron un fuerte impulso al establecimiento de este tipo de pandilla en el norte de Centroamérica (véase Winton, 2007). ${ }^{6}$ En el caso de la legislación reciente en los países centroamericanos afectados por la violencia de las maras, ésta surgió del imaginario popular y de la política puesta en práctica en años recientes, y se

\footnotetext{
${ }^{5}$ Cabe advertir que aunque la palabra "mara" se ha convertido en un nombre genérico para referirse a cierto tipo de pandilla centroamericana, para los jóvenes miembros de ésta no tiene el mismo significado. Ellos se consideran "pandilleros", no "mareros", y pertenecen a una "pandilla", y no a una "mara". La palabra "mara" proviene del nombre de la pandilla: "la Mara Salvatrucha", formada en Los Ángeles por salvadoreños, pero ni siquiera entre los miembros de esta pandilla se usa la palabra "mara" en lugar de "pandilla". El simple hecho de haberles puesto una etiqueta distinta a otras pandillas ha permitido a los medios de comunicación y a los gobiernos de la región destacar su "alteridad" frente a la sociedad.

${ }^{6}$ Un punto interesante es que las maras no han llegado a Nicaragua de igual manera. Más allá de los factores políticos ya mencionados, una explicación parcial podría ser que históricamente los jóvenes nicaragüenses no han migrado a Estados Unidos tanto como sucede en otros países hacia el norte de la región, más bien suelen migrar hacia su país vecino Costa Rica (véase Rocha,
} 


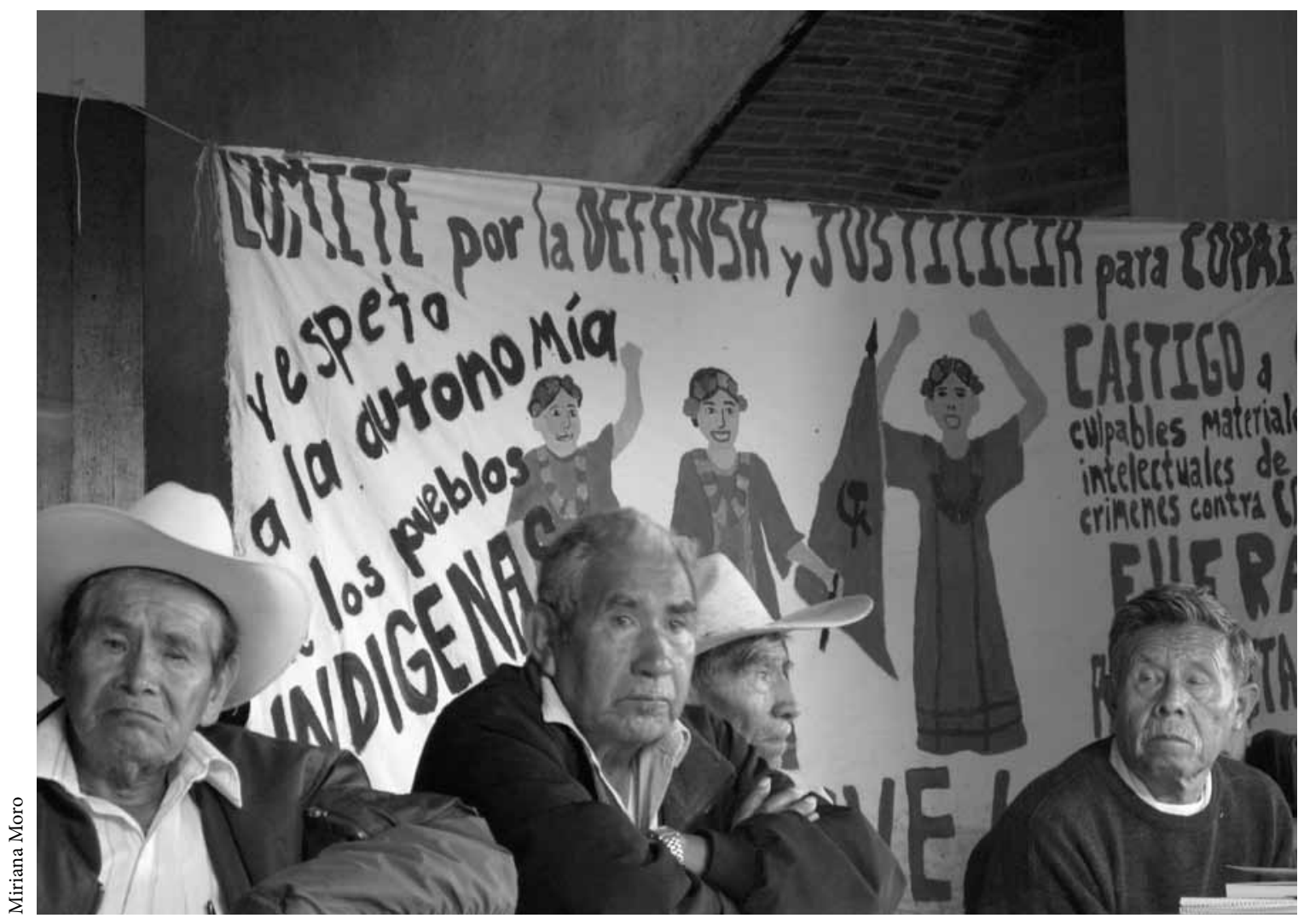

Invitados al Congreso Nacional Indígena, Mezcala, Jalisco, 2010.

relacionaba tanto con los efectos reales de la violencia experimentada en esos países como con la amenaza latente presentada por la diseminación regional del fenómeno de las maras. ${ }^{7}$

Así como la diseminación regional de las pandillas en Centroamérica surge de experiencias comunes de fragmentación social y política, inseguridad y vulnerabilidad, de la misma manera la respuesta formal por parte de los gobiernos se basa en el miedo

2003). Y aunque el segundo destino más común para migrantes nicaragüenses es Estados Unidos, significativamente suelen quedarse en otras regiones del país, por ejemplo Miami, donde el panorama de las pandillas es otro. Hay que destacar que la migración en sí no causa la formación de pandillas, sino que influye de manera considerable sus estructuras y características.

${ }^{7}$ Hasta atravesar el Atlántico: "pandillas latinoamericanas asustan a España”, en <www.eltiempo.com>, 16 de mayo de 2005. e inseguridad que genera la debilidad estatal. Como proponen Huhn et al. (2005), ciertas políticas son una reacción a discursos y no a la violencia en sí, el discurso determina qué reacciones frente a la violencia son consideradas legítimas y cuáles no. La presencia amenazante de pandillas, tanto en el sentido imaginario como real, influye profundamente el discurso de los medios de comunicación y las políticas públicas, lo cual pone en riesgo la misma legitimidad y funcionamiento del Estado y provoca acciones extremas de parte de gobiernos que no hubieran sido necesarias para gobiernos más robustos.

La estrategia regional para enfrentar a las maras, la llamada "legislación antimara" - que en efecto hace ilegal pertenecer a una pandilla- y la concomitante autorización colectiva del uso de violencia en contra de estos jóvenes han causado un aumento 
de casos de brutalidad policiaca hacia presuntos pandilleros - en algunos casos fatales- y frecuentes acusaciones de limpieza social y justicia por mano propia. La represión violenta es permitida, sustentada en un proceso de estigmatización y deshumanización extrema de los pandilleros, que permite la aprobación colectiva de violencia contra estos jóvenes. Algunos comentaristas han llegado a sugerir que las llamadas políticas de "mano dura" representan el comienzo de un apartheid social en El Salvador (Proceso, 2003). Así, políticas antipandillas en Centroamérica representan un elemento cuasi totalitario dentro de supuestas democracias. Bajo tales condiciones se hace posible concebir una situación en la cual un asesinato no sea considerado un crimen en los marcos normativos tanto legales como socioculturales.

De esta manera, las políticas estigmatizantes y represivas tienen un amplio efecto sociocultural, de modo que los efectos de las políticas sobreviven a las políticas mismas, hasta reforzar las divisiones entre los que pertenecen a la nación y los que no (véase Davids, en prensa). Se podría argumentar que esta situación refleja un proceso más global, como comenta Sassen (2007), en la cual muchas ciudades son los sitios de la desvalorización extrema de los desfavorecidos. Es decir, que el actual sistema económico permite que sectores enteros de la humanidad se vuelvan "superfluos", y por tanto desechables.

Por extensión, hay una relación que se autoperpetúa entre el discurso, la legislación y los vínculos entre las pandillas y el crimen organizado. En primer lugar, las políticas de mano dura en Centroamérica, relacionadas con la histeria nacional o hasta regional, tuvieron el efecto secundario no deseado de alinear a las pandillas con el crimen organizado en mayor grado que en el pasado, sobre todo en el narcomenudeo y el transporte de migrantes indocumentados, como se mencionó arriba. El hecho de que últimamente muchas pandillas se hayan "diversificado" significa que han dejado de ser un asunto local del barrio, convirtiéndose en una obsesión regional - a escala transnacional- en años recientes (véase Reguillo, 2005; Zilberg, 2004).
Las políticas represivas agravan aún más la reducción de la capacidad estatal de gobernar efectivamente, al ocultar otras formas insidiosas de violencia y crimen. Además, se ha constatado que aunque los índices de violencia son altos, es un mito que los crímenes con violencia se hayan elevado desproporcionadamente en la década pasada debido al crecimiento de las pandillas, y tampoco es cierto que los jóvenes sean responsables del alto índice de asesinatos. De hecho, los miembros de las pandillas tienen más probabilidades de ser asesinados que de ser asesinos. En efecto, se ha documentado que toda reducción de violencia pandilleril ha sido contrarrestada por un incremento en la muerte de miembros de pandillas a manos de la policía y grupos extremistas de vigilancia civil.

Aunque es importante no perder de vista que el miedo a las pandillas está basado a menudo en experiencias reales, tales políticas hacen que la rehabilitación de los miembros de pandillas sea prácticamente imposible. Los jóvenes que viven como integrantes de una pandilla dependen en general de ella para su supervivencia, tanto económica como social, y sin alternativas seguras ni adecuadas la rehabilitación no sólo les ofrece poco, sino que además representa el gran riesgo de que sean perseguidos simplemente por portar los tatuajes de la pandilla, y ahora no únicamente por parte de otros pandilleros, sino también por la policía y por grupos de limpieza social. De por sí, la mayoría de los pandilleros no sobreviven a la vida pandilleril. Es aun más trágico el hecho de que al tratar de "calmarse" corran el mismo riesgo de persecución o muerte. En palabras de un líder del Barrio 18: "hay algunos que tienen familia, hijos y tal vez trabajan, pero sólo por andar con tatuajes pueden ser golpeados o hasta asesinados".

A veces, la única opción para los miembros de pandillas en tal situación no es dejar de pertenecer a la pandilla, sino transformar su actuación, reduciendo su presencia pública. Históricamente, la presencia y visibilidad física, territorial, les ha sido

\footnotetext{
${ }^{8}$ Entrevista en San Pedro Sula, Honduras, 21 de agosto de 2004.
} 
fundamental, pero ahora parece que la mera razón de su existir está cambiando, y que las pandillas se están convirtiendo en organizaciones clandestinas en mayor medida, lo cual es potencialmente más desestabilizador para el Estado. Estas transformaciones en la estructura de las pandillas podría considerarse como un elemento en la institucionalización de las mismas, en el sentido de que se están insertando de una manera más sistémica en el panorama institucional a nivel local. Como dice Medina y Mateu-Gelabert (2007), han podido desarrollar una identidad criminal más sólida, cimentar su base social y económica, y por tanto su poder. Sin embargo, cabe destacar que para el grueso de los pandilleros involucrarse con un grupo de crimen organizado implica meterse a muy bajo nivel y su clara desventaja. Aunque esta estrategia en teoría les ofrece una ganancia significativa, el hecho de que la mayoría de los pandilleros sigan viviendo en condiciones de pobreza extrema supone que es una estrategia de supervivencia, no de expansión de la pandilla.

$\mathrm{Si}$, como se mencionó antes, las pandillas se establecieron en la ausencia de legitimidad y hegemonía estatal - desafiando la legalidad en términos de confrontar una ausencia, en lugar de una presencia de la misma-, entonces una reacción autoritaria que es efectivamente un intento de "llenar la ausencia de legitimidad con una dosis doble de legalidad" (Reguillo, 2005: 77) es, a fin de cuentas, ineficaz. En primer lugar, "cuando la muerte, la inestabilidad, la incertidumbre, la desesperanza y el desapego se instalan como experiencias cotidianas, el castigo ejemplar es irrelevante" (Reguillo, 2005: 80). En segundo lugar, las encarcelaciones masivas tienen el efecto de convertir las prisiones en una extensión de la vida pandilleril, en una experiencia normalizada (Hagedorn, 2007). Sabemos que la prisión no rehabilita, sólo refuerza las alianzas y los lazos entre pandillas y grupos criminales, y la alienación de estos jóvenes. El encarcelamiento masivo no ayuda a desmantelar pandillas; por el contrario, refuerza y transforma la manera en la que operan. De hecho, se ha argumentado que en el entorno de los centros penales las pandillas han desarrollado estructuras más cerradas, con un nivel mayor de cohesión social, lo cual posiblemente ha contribuido a su institucionalización en la calle y al afianzamiento de este problema social (Medina y Mateu-Gelabert, 2007).

Según la discusión anterior, puede concluirse que son las relaciones entre pandillas, comunidad y Estado las que le dan forma a la estructura, a la función y a los impactos de las pandillas contemporáneas en Centroamérica, y así las pandillas son un fenómeno eminentemente relacional. Las políticas represivas no sólo cambiaron la conducta de las pandillas, pues a su vez desplazaron físicamente a muchos de sus integrantes. Como un portavoz de la Secretaría de Seguridad en Honduras entusiasmadamente comenta: "frente a nuestra ofensiva, miles de miembros de pandillas han huido a otros países de Centroamérica y a los Estados Unidos y México". Entonces, las políticas para contener a las pandillas lograron, paradójicamente, reproducir y transformar sus redes y flujos transnacionales. El más significativo de estos movimientos ha sido la reciente "incursión" de pandillas en el sur de México, donde se dice que la frontera, con todas sus posibilidades criminales, les ha proveído de múltiples oportunidades para sobrevivir. Pero si bien las políticas antipandillas jugaron un rol importante en el establecimiento de las maras en México, esto es sólo parte del problema.

Estas pandillas fueron registradas en el sur de México tras el desplazamiento causado por el huracán Mitch en 1998, y aunque continuaron reforzándose por el derivado de las políticas antipandillas - como sucedió anteriormente en Centroaméri$\mathrm{ca}-$ se convirtieron en una mezcla de influencias culturales externas y panoramas locales de privación, de tal manera que una vez que se encontraban semiestablecidas la mayoría de sus miembros eran mexicanos y ya eran distintas a sus contrapartes centroamericanas. Con todo y que ostensiblemente se conformaban según la estructura y

\footnotetext{
${ }^{9}$ El Universal, 5 de julio de 2005.
} 
prácticas de las maras - causando como resultado un considerable pánico popular y político一, aún durante sus periodos más activos y conflictivos no alcanzaron los niveles de brutalidad testificados en Centroamérica: son pandillas menos extremas tanto en criminalidad como en violencia, ya que el contexto sociocultural mexicano es considerablemente diferente, lo cual reitera la importancia del contexto local a la hora de determinar la índole de las llamadas pandillas "transnacionales".

La respuesta reaccionaria del Estado no ha sido proporcional a la escala real del problema, pues ha respondido de forma exagerada a una amenaza primordialmente imaginaria. ${ }^{10}$ En México, como antes en Centroamérica, los medios de comunicación son responsables de incitar una histeria sobre su potencial propagación, en tal medida que "la Mara Salvatrucha" ha salido a la luz pública, a pesar de que la gran mayoría de la gente no tiene conocimiento ni experiencia de lo que son estas pandillas. El gobierno mexicano, por tanto, optó por emular el enfoque de sus vecinos del sur al encarcelar a miles de jóvenes tatuados. A fin de cuentas, este plan ha reducido significativamente la presencia abierta de las pandillas en las ciudades fronterizas del sur de México, pero esto no ha significado en absoluto el fin del problema. Las pandillas que restan, casi de igual forma que las pandillas en Centroamérica, han cambiado la manera de operar: ya no utilizan tatuajes como antes ni se reúnen en grandes grupos, se han convertido en un "blanco invisible". Además, como la "barrida" se basó más en el miedo, la amenaza o el prejuicio que en hechos reales, resulta que muchos jóvenes encarcelados por pandillerismo no habían cometido crimen alguno.

\footnotetext{
${ }^{10}$ Es interesante considerar por qué, pese a esta menor incursión, las maras no han podido enraizarse en México como lo han hecho en Centroamérica. Especulativamente, hay un factor clave que podría ser de particular relevancia aquí: las pandillas juveniles y las redes del crimen organizado diversas y numerosas se encuentran bien establecidas, y relativamente bien organizadas, así que simplemente no dejan espacio institucional para algún nuevo movimiento de importancia, como el de las maras.
}

Cabe mencionarse, respecto de la reacción del Estado frente a la amenaza de violencia organizada, que a pesar de la presunta reducción en la actividad militar interna con la llegada de la democracia a América Central, los altos niveles de crimen delincuencial han llevado a los gobiernos de toda la región a reclutar la ayuda militar en un intento - aparente- por aumentar la seguridad. Si bien los gobiernos ven con frecuencia la violencia como un reto o una prueba a su autoridad (Saldomando, 1998), una presencia militar intermitente en las calles en años recientes ha sido una herramienta políticamente útil para demostrar "mano fuerte" contra el crimen. Sin embargo, tales políticas complican aún más el ejercicio legítimo de violencia y control, aumentan el número de actores implicados en estas prácticas y además van en contra de normas democráticas establecidas en los acuerdos de paz. Como comenta Mesa (2009: 263): "es esencial prevenir la militarización de la respuesta a los problemas sociales".

\section{CONCLUSIONES}

El presente trabajo ha argumentado que uno de los aspectos más significativos de la violencia contemporánea — sobre todo la urbana - es la amplia gama de instituciones y grupos violentos - estatales, privados, civiles, formales, informales, criminales- $y$, muy significativamente, las conexiones entre éstos en diferentes contextos y su arraigo sistémico. Se ha visto que la institucionalización de distintos grupos violentos en diferentes contextos puede ser un elemento importante en el análisis de violencia contemporánea en la región centroamericana. En Centroamérica, la vulnerabilidad estatal afecta de manera directa su capacidad para responder en forma adecuada a la violencia, igual que a otros problemas sociales. $\mathrm{Al}$ contrario, sus políticas alientan una amplia gama de organizaciones ilícitas y violentas. El hecho de que estos grupos se estén institucionalizando a diferentes niveles y que la línea que los divide se borre cada vez más hoy representa una amenaza importante a 


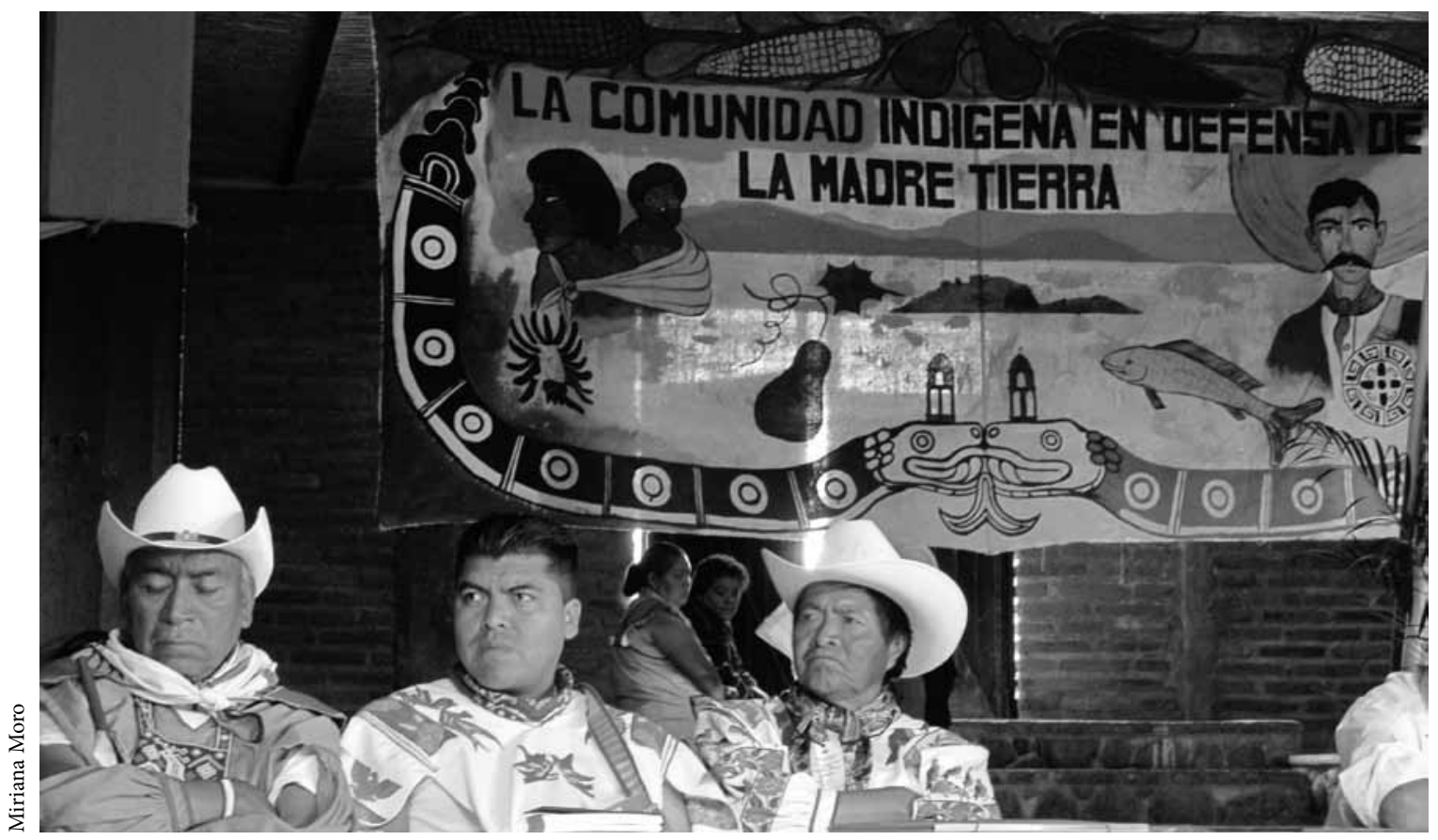

Indígenas participantes en el Congreso Nacional Indígena en Mezcala, Jalisco, 2010.

la estabilidad de la región. No cabe duda de que existe una crisis en el contrato social en estos contextos y muchos grupos violentos y/o ilícitos, incluyendo a las pandillas en mayor o menor grado, se han institucionalizado - siendo esto consolidado y arraigado sistémicamente- como resultado de la falta de legitimidad estatal. Duras y represivas acciones frente a ciertos grupos violentos en Centroamérica, con la colusión de muchas entidades estatales en las mismas redes ilícitas, han creado una verdadera crisis de gobernanza en la región. Como argumenta Cruz (2003), puede ser más relevante hoy hablar sobre los procesos de (re)autorización de Centroamérica, que de su democratización.

\section{Bibliografía}

Briceño-León, Roberto y Verónica Zubillaga, 2002, "Violence and Globalization in Latin America", en Current Sociology, vol. L, núm. 1, pp. 19-37.
Cruz, José Miguel, 1999, “La victimización por violencia urbana: niveles y factores asociados en ciudades de América Latina y España”, en Pan American Journal of Public Health, Special Issue on Violence, vol. V, núm. 4-5, pp. 259-267.

, 2003, "Violencia y democratización en Centroamérica: el impacto del crimen en la legitimidad de los regímenes de posguerra", en América Latina Hoy, núm. 35, pp. 19-59.

Davids, T., en prensa, "Urban Youth, Poverty and Gang Violence in San Salvador: Ambiguities of Security and Violence Between the Local and the Global", en María Eugenia Suárez de Garay y José Carlos G. Aguiar (coords.), La seguridad entre lo local y lo global: los entornos latinoamericanos, Instituto Tecnológico y de Estudios Superiores de Occidente, Centro Universitario de Ciencias Sociales y Humanidades-Universidad de Guadalajara, Centro de Estudios y Documentación Latinoamericanos-Universidad de Ámsterdam, Guadalajara.

Dowdney, Luke, 2003, Children of the Drug Trade: A Case Study of Children in Organised Armed Violence in Rio de Janeiro, 7Letras, Río de Janeiro.

Fernández García, María Cristina, 2004, "Lynching in Guatemala: Legacy of War and Impunity”, monografía de investigación, Harvard University, Cambridge. 
Gaviria, Alejandro, 1998, Increasing Returns and the Evolution of Violent Crime: The Case of Colombia, Discussion Paper, núm. 98-14, Department of Economics, University of California, San Diego.

Hagedorn, J. M., 2007, "Gangs, Institutions, Race and Space: The Chicago School Revisited", en J. M. Hagedorn (ed.), Gangs in the Global City, University of Illinois Press, Urbana y Chicago, pp. 13-33.

Harris, Bronwyn, 2003, "Spaces of Violence, Places of Fear: Urban Conflict in Postapartheid South Africa", ponencia, Conflicts and Urban Violence, Foro Social Mundial Temático, 16-20 de junio, Cartagena, en línea: $<$ http://www.csvr.org.za/docs/urbansafety/englishspaces.pdf $>$.

Huhn, Sebastian, Anika Oettler y Peter Peetz, 2005, "La telaraña de los discursos sobre violencia en Centroamérica”, en Iberoamericana, núm. 19, pp. 188-193.

Kirby, Perpetua, 2006, Vulnerability and Violence: The Impact of Globalization, Pluto Press, Londres.

Kruijt, Dirk y Kees Koonings, 1999, "Introduction: Violence and Fear in Latin America", en Dirk Kruijt y Kees Koonings (eds.), Societies of Fear: The Legacy of Civil War, Violence and Terror in Latin America, Zed, Londres, pp. 1-30.

Leeds, Elisabeth, 1996, "Cocaine and Parallel Politics in Level Democratization", en Latin American Research Review, vol. XXXI, núm. 3, pp. 47-85.

Medina, Juan José y Pedro Mateu-Gelabert, 2007, "Prólogo", en Demoscopia, S. A., Maras y pandillas, comunidad y policía en Centroamérica, Agencia Sueca de Cooperación Internacional para el Desarrollo, Banco Centroamericano de Integración Económica, Guatemala.

Mesa, Manuela, 2009, "Violencia transnacional en Centroamérica: retos y desafíos", en Manuela Mesa (coord.), Crisis y cambio en la sociedad global, Anuario 20092010, Centro de Educación e Investigación para la Paz, Icaria, Barcelona, pp. 247-264.

Moser, Caroline y Cathy McIlwaine, 2004, Encounters with Violence in Latin America: Urban Poor Perceptions from Colombia and Guatemala, Routledge, Londres.

Moser, Caroline y Ailsa Winton, 2002, Violence in the Central American Region: Towards an Integrated Framework for Violence Reduction, documento de trabajo, núm. 171, Overseas Development Institute, Londres.

Pecaut, Daniel, 1999, "From the Banality of Violence to Real Terror: the Case of Colombia", en Dirk Kruijt y Kees Koonings (eds.), Societies of Fear: The Legacy of Civil War, Violence and Terror in Latin America, Zed, Londres, pp. 141-167.

Programa de las Naciones Unidas para el Desarrollo, 1998, Guatemala: los contrastes del desarrollo humano 1998, Programa de las Naciones Unidas para el Desarrollo, Guatemala.

Proceso, 2003, "Plan 'Mano Dura': violencia estatal contra las maras”, en Envío, núm. 258, en línea: <http://www. envio.org.ni/articulo/1283>.

Reguillo, Rossana, 2005, "La mara: contingencia y afiliación con el exceso", en Nueva Sociedad, núm. 200, pp. 70-84, en línea: <http://www.nuso.org/upload/articulos/3297_1.pdf $>$.

Rocha, José Luis, 2003, "Why Do They Go? Theories on the Migration Trend”, en Envío, núm. 264, en línea: $<$ http://www.envio.org.ni/articulo/2105>.

Rodgers, Dennis, 2003, "Youth Gangs in Colombia and Nicaragua: New Forms of Violence, New Theoretical Directions?", en Anders Rudqvist (ed.), Breeding Inequality-Reaping Violence Exploring Linkages and Causality in Colombia and Beyond, Outlook on Development Series, Collegium for Development Studies, Uppsala, pp. 111-141.

— 2006 , "Living in the Shadow of Death: Gangs, Violence and Social Order in Urban Nicaragua 19962002", en Journal of Latin American Studies, vol. XXXVIII, núm. 2, pp. 267-292.

Saldomando, Ángel, 1998, "Violencia e inseguridad en América Central: de la guerra a la gestión cotidiana de la violencia”, en Programa de las Naciones Unidas para el Desarrollo, Violencia en una sociedad en transición, Programa de las Naciones Unidas para el Desarrollo, San Salvador, pp. 72-87.

Sassen, Saskia, 2007, “The Global City: One Setting for New Types of Gang Work and Political Culture?”, en J. M. Hagedorn (ed.), Gangs in the Global City, University of Illinois Press, Urbana y Chicago, pp. 97-119.

Winton, Ailsa, 2004, "Urban Violence: A Guide to the Literature", en Environment and Urbanization, vol. XVI, núm. 2, pp. 165-184. , 2007, "Youth Gangs in North and Central America: From Barrio Gangs to Transnational Syndicates?", ponencia, Workshop on Crime, Law and Governance in Latin America and the United States, Universidad de Florida, 29 de abril-1 de mayo, California.

Zilberg, Elana, 2004, "Fools Banished from the Kingdom: Remapping Geographies of Gang Violence between the Americas (Los Ángeles y San Salvador)", en American Quarterly, vol. LVI, núm. 3, pp. 759-779.

Zúñiga Núñez, Mario, 2007-2008, “Las 'maras' salvadoreñas como problema de investigación para las ciencias sociales", en Anuario de Estudios Centroamericanos, núm. 33-34, pp. 87-110. 